\title{
Omega-3 Fatty Acids Improve Recovery, whereas Omega-6 Fatty Acids Worsen Outcome, after Spinal Cord Injury in the Adult Rat
}

\author{
Von R. King, ${ }^{\star}$ Wenlong L. Huang, ${ }^{\star}$ Simon C. Dyall, Olimpia E. Curran, John V. Priestley, and Adina T. Michael-Titus \\ Neuroscience Centre, Institute of Cell and Molecular Science, St. Bartholomew's and the Royal London School of Medicine and Dentistry, Queen Mary \\ University of London, London E1 2AT, United Kingdom
}

\begin{abstract}
Spinal cord injury (SCI) is a cause of major neurological disability, and no satisfactory treatment is currently available. Evidence suggests that polyunsaturated fatty acids (PUFAs) could target some of the pathological mechanisms that underlie damage after SCI. We examined the effects of treatment with PUFAs after lateral spinal cord hemisection in the rat. The $\omega$-3 PUFAs $\alpha$-linolenic acid and docosahexaenoic acid (DHA) injected $30 \mathrm{~min}$ after injury induced significantly improved locomotor performance and neuroprotection, including decreased lesion size and apoptosis and increased neuronal and oligodendrocyte survival. Evidence showing a decrease in RNA/DNA oxidation suggests that the neuroprotective effect of $\omega$-3 PUFAs involved a significant antioxidant function. In contrast, animals treated with arachidonic acid, an $\omega-6$ PUFA, had a significantly worse outcome than controls. We confirmed the neuroprotective effect of $\omega-3$ PUFAs by examining the effects of DHA treatment after spinal cord compression injury. Results indicated that DHA administered 30 min after spinal cord compression not only greatly increased survival of neurons but also resulted in significantly better locomotor performance for up to 6 weeks after injury.

This report shows a striking difference in efficacy between the effects of treatment with $\omega-3$ and $\omega-6$ PUFAs on the outcome of SCI, with $\omega$-3 PUFAs being neuroprotective and $\omega$-6 PUFAs having a damaging effect. Given the proven clinical safety of $\omega-3$ PUFAs, our observations show that these PUFAs have significant therapeutic potential in SCI. In contrast, the use of preparations enriched in $\omega$ - 6 PUFAs after injury could worsen outcome after SCI.
\end{abstract}

Key words: spinal cord injury; neuroprotection; omega-3 fatty acids; apoptosis; inflammation; oxidation

\section{Introduction}

The devastating effects of spinal cord injury (SCI) are a result of not only the initial injury but also a process of spreading secondary degeneration that affects both local neurons and glia, and en passant axons. Several pathophysiological mechanisms underlie this degeneration, including excitotoxicity (Hall and Braughler, 1986) and inflammation triggered by macrophages and microglia that release inflammatory cytokines (Profyris et al., 2004). Although numerous treatments have targeted these pathogenetic processes in preclinical studies (Leker and Shohami, 2002), most have had limited success in clinical trials. One of the reasons for this may be the complexity of the secondary degenerative response. Many treatments affect only one aspect of this response, and a successful treatment will probably have to target several of these mechanisms.

Over the past 10 years, there has been increased interest in the

\footnotetext{
Received Dec. 25, 2005; revised March 13, 2006; accepted March 22, 2006.

This work was supported by the Wellcome Trust, St. Bartholomew's and The Royal London Charitable Foundation, and Corporate Action Trust.

*V.R.K. and W.L.H. contributed equally to this work

Correspondence should be addressed to Dr. Von R. King, Neuroscience Centre, Institute of Cell and Molecular Science, 4 Newark Street, London E1 2AT, UK. E-mail: vonrking@yahoo.co.uk. DOI:10.1523/JNEUROSCI.5539-05.2006

Copyright $\odot 2006$ Society for Neuroscience $\quad$ 0270-6474/06/264672-09\$15.00/0
}

health benefits of polyunsaturated fatty acids (PUFAs), with evidence emerging that $\omega$-3 PUFAs have significant therapeutic potential in neurology and psychiatry (Lauritzen et al., 2000; Emsley et al., 2003). PUFAs are structural components of phospholipids, which are the main constituents of cell membranes. PUFAs of the $\omega$-3 series include $\alpha$-linolenic acid (ALA), eicosapentaenoic acid (EPA) and docosahexaenoic acid (DHA), the latter two being the longer chain compounds, whereas ALA is the biosynthetic precursor. Recent evidence shows that $\omega-3$ PUFAs can modulate several of the processes that contribute to the secondary degeneration in the CNS. For example, $\omega-3$ PUFAs have antioxidant effects (Sarsilmaz et al., 2003) as well as anti-inflammatory effects through inhibition of the production of proinflammatory cytokines (Endres and von Schacky, 1996). PUFAs can also block apoptosis (Martin et al., 2002), and several studies have documented their neuroprotective effects in vitro and in vivo (Blondeau et al., 2002; Wang et al., 2003). These observations suggest that $\omega-3$ PUFAs could have significant therapeutic potential in the treatment of SCI.

The present study examined the effects of PUFA treatment in a rat model of SCI. The $\omega-3$ PUFAs ALA and DHA were injected 30 min after a spinal cord hemisection, and their effects were studied on hindlimb locomotor function, lesion size, cell survival, and apoptosis. To begin to determine the mechanism of any 
neuroprotective effect of PUFA treatment, we also examined the degree of RNA/DNA oxidation, a process shown to be present in both neurodegenerative diseases and after traumatic CNS injury (Facheris et al., 2004). To establish whether any neuroprotective effects were specific for $\omega$-3 PUFAs, we also examined the effects of the $\omega-6$ PUFA arachidonic acid (AA) and the $\omega-9$ monounsaturated fatty acid oleic acid (OA). AA and OA have complex profiles and have been shown to induce both neuroprotection and neurodegeneration (Katsuki and Okuda, 1995; Toborek et al., 1999; Rodriguez-Rodriguez et al., 2004), depending on the experimental model. In addition, to further confirm the efficacy of $\omega-3$ PUFAs after spinal cord injury, we examined the effects of DHA treatment after spinal cord compression injury on neuronal survival as well as hindlimb locomotor recovery.

\section{Materials and Methods}

Animals and surgical procedures. Spinal cord hemisection and perfusion were performed as described previously (King et al., 2004). All animal procedures were approved by the United Kingdom Home Office. Adult male Wistar rats (200-250 g) were anesthetized using 2-3\% halothane, the spinal cord between the T7 and T9 levels was exposed by laminectomy, and a hemisection extending from the midline to the lateral edge of the spinal cord was made with iris scissors. The lesion site was covered with gelfoam, and the overlying muscle and skin were sutured. Thirty minutes after hemisection, animals received a tail vein injection of vehicle $(n=8)$ or one of the following fatty acids at a dose of $250 \mathrm{nmol} / \mathrm{kg}$, in a volume of $5 \mathrm{ml} / \mathrm{kg}$ : the $\omega-3$ PUFAs ALA $(n=5)$ or DHA $(n=10)$, the $\omega$-6 PUFA AA $(n=5)$, or the $\omega-9$ monounsaturated fatty acid OA $(n=$ 10). Fatty acids were dissolved at $1 \mathrm{M}$ in ethanol and then diluted with physiological saline, and solutions were adjusted to $\mathrm{pH}$ 7.4. All animals survived for 1 week after surgery.

A second group of animals received a static compression injury of the spinal cord as described previously (Nystrom et al., 1988; Huang et al., 2006). Briefly, 22 animals were anesthetized using 2-3\% halothane, and the spinal cord at the T12 level was exposed by laminectomy. The T11 and T13 spinal processes were then clamped to prevent movement of the spinal cord. A compression plate (area, $2 \times 5 \mathrm{~mm}$ ) that was suspended from a platform via a metal rod was then placed on the dura of the exposed spinal cord, and a $50 \mathrm{~g}$ weight was placed on the platform for 5 min. The lesion site was then closed (see above). Thirty minutes after compression, animals received a tail vein injection of either vehicle or DHA (see details above). The bladders of the animals were manually voided twice a day for the first week and once daily thereafter until the establishment of reflex voiding. Animals survived for either $7 \mathrm{~d}$ (vehicle, $n=5$; DHA, $n=5$ ) or 6 weeks (vehicle, $n=6$; DHA, $n=6$ ).

At the appropriate postsurgical interval, animals were deeply anesthetized with sodium pentobarbital $(50 \mathrm{mg} / \mathrm{kg}$, i.p.) and perfused through the ascending aorta with $4 \%$ paraformaldehyde in $0.1 \mathrm{~m}$ phosphate buffer, $\mathrm{pH}$ 7.4. The spinal cord was dissected out, postfixed in $4 \%$ paraformaldehyde for $1-2 \mathrm{~h}$, and cryoprotected in $15 \%$ sucrose, and then 10 $\mu \mathrm{m}$ horizontal cryostat sections were cut through the hemisection site. For animals that received static compression injury, transverse sections through the entire rostrocaudal extent of the lesion site were taken.

Histochemistry. Sections were processed using standard immunofluorescence procedures to the following antisera: mouse neuronal nuclear antigen (NeuN) (1:200; Chemicon, Harrow, UK) to label neurons, mouse adenomatous polyposis coli tumor suppressor protein (APC) (1: 200; Calbiochem, La Jolla, CA) to label oligodendrocytes, mouse glial fibrillary acid protein (GFAP) (1:1000; Chemicon) to label astrocytes, mouse ED1 (1:1000; Serotec, Oxford, UK) to label macrophages, and mouse 8-hydroxyguanosine ( $\mathrm{oh}^{8} \mathrm{G}$ ) (1:1000; QED Biotech, San Diego, CA) to label cells undergoing RNA/DNA oxidation. After $48 \mathrm{~h}$ of incubation in primary antibody and washes in PBS, slides were incubated for $2 \mathrm{~h}$ in secondary antibody conjugated to tetramethylrhodamine isothiocyanate (1:400; Stratech Scientific, Cambridgeshire, UK) and then either coverslipped in PBS glycerol or processed for terminal deoxynucleotidyl transferase-mediated biotinylated UTP nick end labeling (TUNEL) using a terminal transferase-mediated dUTP nick end labeling kit (Promega, Southampton, UK). This involved a $1 \mathrm{~h}$ incubation in biotinylated dUTP and terminal deoxynucleotidyl transferase, followed by a $2 \mathrm{~h}$ incubation in avidin extra fluorescein isothiocyanate (1:600; Sigma, Poole, UK).

To assess the effects on spinal tracts, sections were processed with the myelin stain Luxol fast blue. Sections were incubated at $37^{\circ} \mathrm{C}$ overnight in $0.1 \%$ Luxol fast blue (Sigma) in 95\% ethanol and 0.05\% glacial acetic acid and then differentiated in $1 \%$ lithium carbonate followed by $70 \%$ ethanol. Sections were then rinsed in tap water, dehydrated, and coverslipped.

Behavioral assessment. Hindlimb locomotor function for animals that received a spinal cord hemisection was assessed 1, 3, 5, and $7 \mathrm{~d}$ after surgery in animals that received spinal cord hemisection. For the week before surgery, animals were habituated and trained on all behavioral tasks. The tasks used were as follows: (1) beam walk, (2) horizontal ladder, and (3) open field. The beam walk test was similar to that described by Metz et al. (2000). Animals were scored on their ability to traverse 1.2-m-long wooden beams of three different widths $(4.5,3.5$, and $2.5 \mathrm{~cm}$ ). The following scoring system was used: 0 for traversing less than half the beam, 0.5 for traversing half the length of the beam, 1 for traversing the whole beam, 1.5 for traversing the whole beam and showing at least some stepping with the hindlimb ipsilateral to the lesion, and 2 for traversing the whole beam with accurate foot placement of the hindlimb ipsilateral to the lesion. Animals that scored a zero on any beam were not tested on narrower beams and were assigned a score of zero for these beams. A maximum score of six was possible. In the horizontal ladder test, animals were assessed on the number of footslips made when crossing a $1-\mathrm{m}$-long horizontal ladder $(11 \mathrm{~cm}$ wide with $5 \mathrm{~cm}$ between each rung). A footslip was defined as a failure to at least partially grasp an individual rung with the hindlimb ipsilateral to the lesion. In the open field test, animals were placed in an open field and rated on a 21-point scale for the use of the hindlimb ipsilateral to the side of the lesion, using the Basso, Beattie, Bresnahan scale (BBB score) (Basso et al., 1996). Animals that received spinal cord compression and survived for 6 weeks were assessed at $1 \mathrm{~d}$, 1 week, 4 weeks, and 6 weeks after surgery on this test.

Image and data analysis. The sections were viewed on a Leica (Wetzlar, Germany) epifluorescence microscope using Y3 (tetramethylrhodamine isothiocyanate) or L4 (fluorescein isothiocyanate) filter blocks. Images were taken using a Hamamatsu (Herrsching, Germany) C4742-95 digital camera and HiPic software (Hamamatsu). Figures were prepared using Adobe Photoshop (Adobe Systems, San Jose, CA).

Quantitative analysis for the number of TUNEL-positive nuclei was conducted by capturing an image of TUNEL labeling in the intact tissue immediately rostral to the hemisection site. All TUNEL-positive nuclei within the area were then counted in a $500 \times 500 \mu \mathrm{m}$ measuring frame. An identical procedure was conducted in the intact tissue immediately caudal to the hemisection site. For each animal, a measure was taken in a section through the dorsal horn, a section which included the central canal and a section through the ventral horn. The cell counts in the six sections were then used to determine the total number of TUNELlabeled cells per squared millimeter, measured around the lesion area for each animal. Identical analyses were conducted for the number of NeuNlabeled and APC-labeled cells. A similar analysis was conducted for $\mathrm{oh}^{8} \mathrm{G}$-labeled cells, with the exception that only sections through the dorsal horn and ventral horn were counted. In addition, for each animal, an estimate of the lesion size was made by tracing the area within the borders of the lesion as defined by GFAP labeling (see Fig. 1) through a section that included the central canal. A similar quantitative analysis of NeuN-labeled cells was conducted in animals that received spinal cord compression. Briefly, all NeuN-labeled cells within a $100 \times 100 \mu \mathrm{m}$ measuring frame placed within the dorsal and ventral horns on both the left and right sides were counted. Identical measures were taken from six equally spaced sections throughout the center of the lesion for each ani$\mathrm{mal}$, and the number of NeuN-labeled cells per squared millimeter was determined.

For all quantitative analysis of anatomical data, measures were made with the experimenter blind to the animals' treatment group. For each of the above measures, an ANOVA was conducted comparing the treat- 
ment groups, followed by post hoc analysis when appropriate. For behavioral data, twoway repeated-measures ANOVA were conducted comparing the treatment groups over the number of test sessions for a particular behavioral task, followed by post hoc analysis when appropriate.

\section{Results \\ Spinal cord hemisection \\ Lesion size}

Seven days after hemisection injury, the lesion site appeared as an area devoid of GFAP staining, which extended from the midline to the lateral edge of the spinal cord (Fig. $1 A-C$ ). The lesion extent was visibly smaller in DHA- and ALA-treated animals compared with OA- and vehicle-treated animals (Fig. $1 A, B)$. In addition, AA-treated animals had larger lesions than the other four groups, and in the case of two animals showed "islands" of damage that were discontinuous with the original lesion site (Fig. 1C). Quantitative analysis confirmed that DHAand ALA-treated animals had significantly smaller lesions than either OA- or vehicle-treated animals (all comparisons, $p<$ 0.05), and that AA-treated animals (Fig. 1C) had significantly larger lesions than all other groups (Table 1).

Apoptosis

TUNEL-labeled nuclei (Fig. $2 A$ ) were clearly visible, and counterstaining with 4',6-diamidino-2-phenylindole (Fig. 2B) confirmed chromatin condensation and fragmentation in the nuclei of TUNEL-labeled cells. TUNEL labeling was present in all groups at $7 \mathrm{~d}$ after hemisection injury, with the vast majority of apoptotic nuclei present within $500 \mu \mathrm{m}$ of the lesion site. Quantitative analysis showed that DHA- and ALA-treated animals had significantly fewer (30-40\%) TUNEL-labeled cells than OA-, AA-, and vehicle-treated animals (all comparisons, $p<0.05$ ) (Table 1). In contrast, AA treatment resulted in significantly more TUNEL-labeled nuclei than control animals $(p<0.05)$. Double labeling using immunofluorescence to label neurons (Fig. 2C, NeuN) or oligodendrocytes (Fig. 2D, APC) showed that approximately equal percentages of these cell types had apoptotic nuclei, although most TUNEL-labeled cells were not APC or NeuN immunoreactive. In contrast, no astrocytes (GFAP-labeled cells) were seen with TUNEL-positive nuclei. It is not clear in what type of cells the remaining TUNEL-positive nuclei were present. However, it is possible that a loss of antigencity in dying oligodendrocytes or neurons prevented double labeling from occurring in some cells that were actually undergoing apoptosis.

\section{$o h^{8}$ G labeling}

$\mathrm{oh}^{8} \mathrm{G}$ immunoreactivity appeared as punctate cytoplasmic labeling, with little or no labeling within the nucleus itself (Fig. 3). Few labeled cells were seen in the white matter. This, combined with the morphological appearance of labeled cells, suggests that most $\mathrm{oh}^{8} \mathrm{G}$-positive cells were neurons. DHA- and ALA-treated animals had visibly fewer oh ${ }^{8} \mathrm{G}$-positive cells (Fig. $3 B$ ) than either the control (Fig. $3 A$ ) or OA-treated animals. In contrast, AA treatment resulted in a clear increase in $\mathrm{oh}^{8} \mathrm{G}$ labeling (Fig. $3 C$, Table 1) compared with the other groups. This increase was seen not only adjacent to the lesion site but also at several millimeters from the lesion, in an area where oh ${ }^{8} \mathrm{G}$ labeling was not present in the other groups. ANOVA confirmed significant differences between the PUFA-treated groups in the number of oh $^{8} \mathrm{G}$-labeled cells adjacent to the lesion site in both the dorsal and ventral horns (both comparisons, $p<0.001$ ). Subsequent post hoc analysis showed that in the ventral horn, there were fewer oh ${ }^{8} \mathrm{G}$ labeled cells in the DHA- and ALA-treated groups compared with the other groups, whereas AA-treated animals had significantly more labeled cells than all other groups (all comparisons, $p<$ 0.05). Similar results were seen in the dorsal horn, with the exception that ALA-treated animals were not significantly different from OA-treated or control animals in the number of $\mathrm{oh}^{8} \mathrm{G}$ labeled cells.

\section{Neurons}

Seven days after hemisection injury, the number of NeuN-labeled nuclei (Fig. $4 A-C$ ) present in the intact tissue adjacent to the lesion site was greater in ALA- and DHA-treated animals compared with vehicle-, AA-, and OA-treated animals. This difference was most pronounced in tissue immediately adjacent to the lesion site, where numerous NeuN-labeled cells were present in ALA- and DHA-treated animals, but very few labeled cells were present in the remaining groups. Quantitative analysis confirmed these differences, with significantly more NeuN-labeled neurons after DHA and ALA treatment compared with the remaining groups (all comparisons, $p<0.05$ ) (Table 1). In addition, AAtreated animals had significantly fewer NeuN-labeled cells than vehicle controls $(p<0.05)$ and also appeared to have smaller neurons than those in the other groups.

\section{Oligodendrocytes and myelin}

Analysis of APC labeling showed a similar effect to that for NeuN labeling, with DHA- and ALA-treated animals clearly having more APC-labeled oligodendrocytes than vehicle-, OA-, and AAtreated animals (Fig. $4 D-F$ ). Quantitative analysis confirmed that DHA- and ALA-treated animals had significantly more APC-labeled oligodendrocytes than these groups (all comparisons, $p<0.05$ ) (Table 1 ) and also showed that AA-treated animals had significantly fewer APC-labeled cells than all other groups (Table 1) (all comparisons, $p<0.05$ ). The protective effect of ALA and DHA on oligodendrocytes was also reflected in the results obtained with the myelin stain, Luxol fast blue (Fig. 5). Although myelin in the white matter ipsilateral to the lesion showed degeneration in all groups (Fig. $5 A, B$ ) compared with the corresponding white matter contralateral to the lesion (Fig. $5 C$ ), myelin ipsilateral to the lesion in DHA-treated (Fig. 5D) and ALA-treated animals clearly showed less vacuolization and disruption compared with the other three groups (Fig. $5 E$ ). This decreased degeneration was seen several millimeters from the site of injury. 
Table 1. Effects of PUFA treatment on lesion size and cell survival $7 \mathrm{~d}$ after hemisection injury

\begin{tabular}{|c|c|c|c|c|c|}
\hline & $\begin{array}{l}\text { Vehicle } \\
\text { (saline) }\end{array}$ & $\begin{array}{l}\text { ALA } \\
\text { (omega-3 PUFA) }\end{array}$ & $\begin{array}{l}\text { DHA } \\
\text { (omega-3 PUFA) }\end{array}$ & $\begin{array}{l}\text { OA } \\
\text { (omega-9 MUFA) }\end{array}$ & $\begin{array}{l}\text { AA } \\
\text { (omega-6 PUFA) }\end{array}$ \\
\hline Lesion size $\left(\mathrm{mm}^{2}\right)$ & $0.52 \pm 0.06$ & $0.30 \pm 0.03^{a}$ & $0.32 \pm 0.05^{a}$ & $0.64 \pm 0.05$ & $0.86 \pm 0.05^{b}$ \\
\hline TUNEL plus nuclei (per mm²) & $8.67 \pm 0.91$ & $6.51 \pm 0.87^{a}$ & $5.42 \pm 0.73^{a}$ & $9.57 \pm 1.03$ & $11.21 \pm 1.21^{c}$ \\
\hline NeuN plus cells (per mm²) & $60.42 \pm 4.26$ & $81.47 \pm 6.68^{a}$ & $88.87 \pm 3.54^{a}$ & $58.15 \pm 3.23$ & $49.33 \pm 2.37^{c}$ \\
\hline APC plus cells (per mm²) & $35.78 \pm 1.47$ & $41.76 \pm 1.29^{a}$ & $39.74 \pm 2.40^{a}$ & $33.28 \pm 2.44$ & $29.70 \pm 1.49^{c}$ \\
\hline ED1, within lesion (\%) & $17.72 \pm 3.79$ & $13.72 \pm 4.31$ & $19.41 \pm 4.01$ & $16.89 \pm 2.98$ & $28.62 \pm 4.91$ \\
\hline ED1, outside lesion (\%) & $33.67 \pm 5.57$ & $28.91 \pm 4.91$ & $36.72 \pm 6.02$ & $29.71 \pm 4.48$ & $41.22 \pm 7.09^{b}$ \\
\hline $\mathrm{oh}^{8} \mathrm{G}$ plus cells, dorsal (per mm²) & $14.70 \pm 0.82$ & $10.90 \pm 1.20$ & $8.30 \pm 0.82^{a}$ & $14.40 \pm 2.30$ & $22.50 \pm 2.50^{b}$ \\
\hline $\mathrm{oh}^{8} \mathrm{G}$ plus cells, ventral (per $\mathrm{mm}^{2}$ ) & $10.10 \pm 0.76$ & $5.80 \pm 0.62^{a}$ & $5.30 \pm 0.64^{a}$ & $10.60 \pm 1.60$ & $15.20 \pm 2.36^{b}$ \\
\hline
\end{tabular}

${ }^{a}$ Significantly different from vehicle-, AA-, and 0 A-treated animals $(p<0.05)$.

${ }^{b}$ Significantly different from all other groups $(p<0.05)$.

'Significantly different from vehicle-, DHA-, and ALA-treated animals ( $p<0.05$ ).
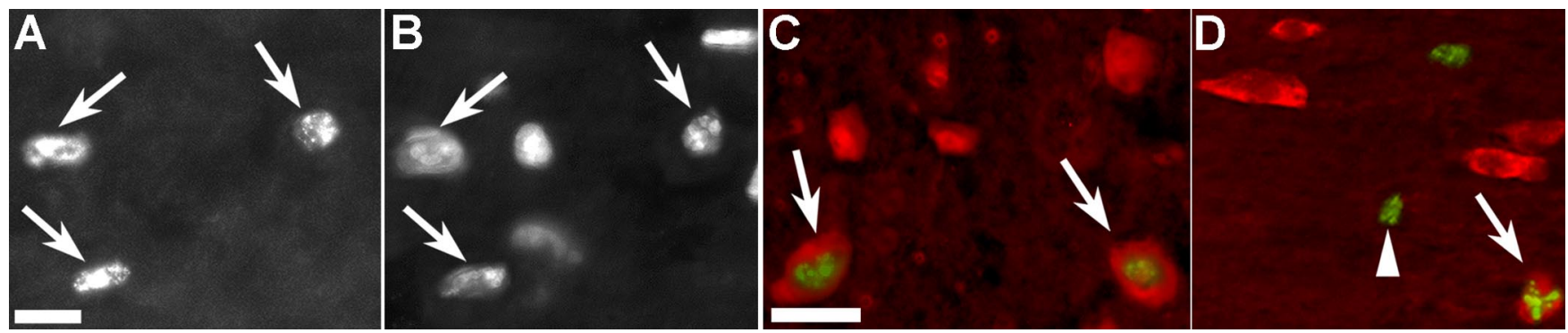

Figure 2. TUNEL labeling of apoptotic nuclei adjacent to the spinal cord hemisection site after a single intravenous injection of either saline or PUFAs after $7 \mathrm{~d}$ of survival. TUNEL-labeled nuclei $(\boldsymbol{A})$ were clearly identifiable in all treatment groups and were confirmed by counterstaining with the nuclear stain 4',6-diamidino-2-phenylindole (B) (corresponding arrows in $\boldsymbol{A}$ and $\boldsymbol{B}$ show double-labeled nuclei). Double labeling ( $\boldsymbol{C}$ for TUNEL (green) and NeuN (red) showed occasional apoptotic nuclei within neurons (arrows). Similarly, double labeling (D) for APC (red) and TUNEL (green) showed occasional double-labeled oligodendrocytes (arrow). Numerous TUNEL single-labeled cells were also present (arrowhead). Scale bars: $A-D, 50 \mu \mathrm{m}$.
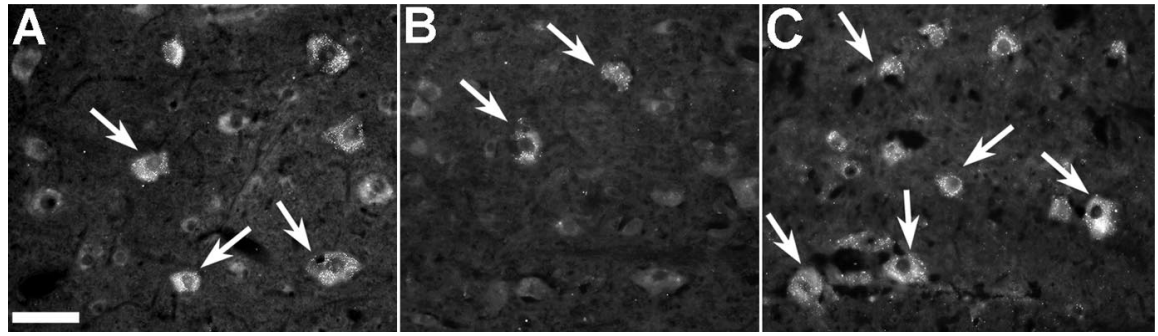

Figure 3. $\quad$ o ${ }^{8} \mathrm{G}$ labeling of RNA/DNA oxidation in cells adjacent to the spinal cord hemisection site after a single intravenous injection of either saline or PUFAs after $7 \mathrm{~d}$ of survival. The number of oh ${ }^{8} \mathrm{G}$-labeled neurons in the area immediately adjacent to the lesion site in vehicle-treated $(\boldsymbol{A})$ and $0 \mathrm{~A}$-treated (not pictured) animals was similar. Both of these groups had noticeably more ${ }_{0}{ }^{8} \mathrm{G}$-labeled cells than either DHA-treated $(\boldsymbol{B})$ and ALA-treated (not pictured) animals, which had few oh ${ }^{8} \mathrm{G}$-labeled cells. In contrast, AA-treated animals $(\boldsymbol{C})$ had substantially more oh ${ }^{8} \mathrm{G}$-labeled cells than all other groups. Scale bar, $50 \mu \mathrm{m}$.

\section{Macrophages}

Examination of ED1 labeling indicated that there was no difference in the density of ED1-positive macrophages within the lesion site for any of the treatment groups (Table 1). However, AA-treated animals had a higher density of ED1 labeling in the intact tissue adjacent to the lesion, compared with the other four groups (all comparisons, $p<0.05$ ) (Table 1), and this increase was apparent as far as $2-3 \mathrm{~mm}$ from the lesion site.

\section{Locomotor behavior}

All animals obtained maximum scores for each task (21 on the $\mathrm{BBB}, 6$ on the beam walk, and 0 errors on the horizontal ladder) during the 3-4 d of training and habituation before receiving injury. Postsurgically, DHA- and ALA-treated animals performed significantly better than the remaining groups on all three behavioral tests during the latter days of testing (days 5-7).

In the open field, at least some plantar stepping was present in the majority of DHA- and ALA-treated animals by the seventh day after surgery, whereas plantar stepping was present in no more than two animals in each of the vehicle-, OA-, or AA-treated animals. Statistical analysis confirmed that ALA- and DHA-treated animals had significantly greater scores on ratings of hindlimb locomotor use in the open field BBB score (Basso et al., 1996) than the remaining three groups $(p<$ 0.05 ) during the third, fifth, and seventh day after surgery (all comparisons, $p<$ 0.05 ), whereas the vehicle-, OA-, and AAtreated groups did not differ at any time point (Fig. 6A).

ALA- and DHA-treated animals also performed better on the beam walk task, with all but two animals from each of these groups able to walk completely across the $3.5 \mathrm{~cm}$ beam and a majority of animals in each of these groups able to walk completely across the $2.5-\mathrm{cm}$ wide (narrowest) beam by $7 \mathrm{~d}$ after surgery. In contrast, fewer than half of the animals in each of the vehicle-, OA-, and AAtreated groups were able to cross completely the 3.5-cm-wide beam, with at least one animal in each group unable to walk completely across the widest beam $(4.5 \mathrm{~cm})$ by the seventh day after surgery. Statistical analysis showed that animals in the ALAand DHA-treated groups had significantly better scores on the beam walk task during the fifth and seventh days after surgery than the vehicle-, OA-, and AA-treated groups (all comparisons, $p<0.05$ ) (Fig. $6 B$ ). The three latter groups were similar at all time points.

Results from the horizontal ladder showed that although both ALA and DHA significantly decreased the number of footslips of the hindpaw ipsilateral to the lesion, DHA had a slightly greater 
effect than ALA, resulting in fewer footslips than all other groups (including the ALA group) at $5 \mathrm{~d}$ after surgery $(p<$ 0.05), whereas both the ALA and DHA groups had fewer ipsilateral footslips than the remaining three groups at $7 \mathrm{~d}$ after surgery $(p<0.05)$ (Fig. 6C). Analysis of foot slips of the hindlimb contralateral to the lesion showed a different pattern (Fig. 6D). Most notably, DHA but not ALA resulted in significantly fewer hindlimb slips compared with vehicle controls (all comparisons, $p<0.05$ ). In addition, this improvement was seen earlier (at the first and third days after surgery) than in the other measures of locomotor function. Last, AA resulted in significantly worse performance than not only the DHA- and ALA-treated groups but also the vehicle control group at 1,3 , and $5 \mathrm{~d}$ after surgery $(p<0.05)$.

\section{Spinal cord compression injury \\ Neurons}

Examination of NeuN labeling after spinal cord compression confirmed the neuroprotective effects of DHA treatment seen after spinal cord hemisection. Specifically, DHA-treated animals clearly had more NeuN-labeled cells than saline-treated animals in all areas of the gray matter at both 1 week (Fig. $7 A, B$ ) and 6 weeks after injury (Fig. 7C,D). Quantitative analysis (Fig. 7E) confirmed that control (saline-treated) compression injury animals had significantly fewer NeuN-labeled cells at 1 week $(56 \%$ decrease) and 6 weeks (66\% decrease) after surgery compared with DHA-treated animals ( $p$ values $<0.05$ ).

\section{Locomotor behavior}

On the first day after surgery, the DHA- and saline-treated animals did not have significantly different BBB scores (Fig. 8), with all animals in both groups showing little or no movement of either hindlimb. By 1 week after surgery, although both DHA and saline-treated animals had improved hindlimb locomotor function, DHA-treated animals had clearly shown more improvement with significantly higher open field ratings $(>3$ points on the BBB scale) than saline-treated animals $(p<0.05)$. By 6 weeks after surgery, although hindlimb locomotor performance of both DHA- and saline-treated animals had improved, animals treated with DHA maintained significantly better hindlimb locomotor performance compared with saline-treated controls $(p<0.05)$.

\section{Discussion}

This study demonstrates for the first time that $\omega$-3 PUFAs are neuroprotective in traumatic SCI and shows that, in contrast, exposure to $\omega$-6 PUFAs in the aftermath of injury could significantly worsen outcome. ALA or DHA treatment 30 min after hemisection of the spinal cord resulted in decreased lesion size, reduced apoptotic cell death, increased neuronal and oligodendrocyte survival, and improved locomotor function. The efficacy of $\omega$-3 PUFA treatment was confirmed in a compression model of spinal cord injury, with DHA treatment resulting in increased neuronal survival, as well as improved locomotor performance as long as 6 weeks after surgery. In striking contrast, AA exacerbated damage and led to worse locomotor performance. Each of these aspects will be briefly discussed, together with the possible mechanisms underlying the neuroprotection and the implications for clinical use of PUFAs in SCI.

Lesion size after hemisection SCI was reduced by $40-45 \%$ by DHA and ALA injection. The reduction in lesion size is likely to be partly attributable to decreased cell death, a conclusion consistent with our finding of increased numbers of NeuNimmunoreactive neurons and APC-immunoreactive oligodendrocytes in the perilesional area. Although loss of immunoreactivity does not necessarily indicate cell death (Unal-Cevik et al., 2004), TUNEL labeling confirmed that both neurons and oligodendrocytes underwent apoptosis and that the $\omega-3$ PUFAs reduced apoptotic death by 25\% (ALA) and 38\% (DHA). Cell death after SCI involves both necrosis and apoptosis, with necrosis prominent at the lesion epicenter and extensive oligodendrocyte apoptosis in degenerating white matter tracts (Yong et al., 1998; Beattie et al., 2000). Although we did not assess necrosis, the decreased lesion size indicates that necrotic cell death is also likely to have been reduced. These protective effects are comparable with the observations of Lang-Lazdunski et al. (2003), who showed that apoptosis induced in the spinal cord after prolonged ischemia can be decreased by an injection of ALA at the onset of reperfusion.

Several possible mechanisms may underlie the reduced neuronal death that we observed. The decreased $\mathrm{oh}^{8} \mathrm{G}$ staining after ALA and DHA treatment suggests that a reduction in oxidative stress, an immediate consequence of SCI (Hall and Braughler, 1986; Xu et al., 2005) that leads to dysfunction of mitochondria and subsequent neuronal death (Sullivan et al., 2005), is involved in the neuroprotective effects of ALA and DHA. This possibility is supported by studies showing that DHA increases the activity of antioxidant enzymes such as glutathione peroxidase and glutathione reductase in hippocampal cells in culture (Wang et al., 2003 ), and that $\omega$-3 PUFAs enhance in vivo the activity of antioxidant systems in the CNS (Sarsilmaz et al., 2003; Songur et al., 2004). In addition, $\omega-3$ PUFAs are not only neuroprotective in rat 


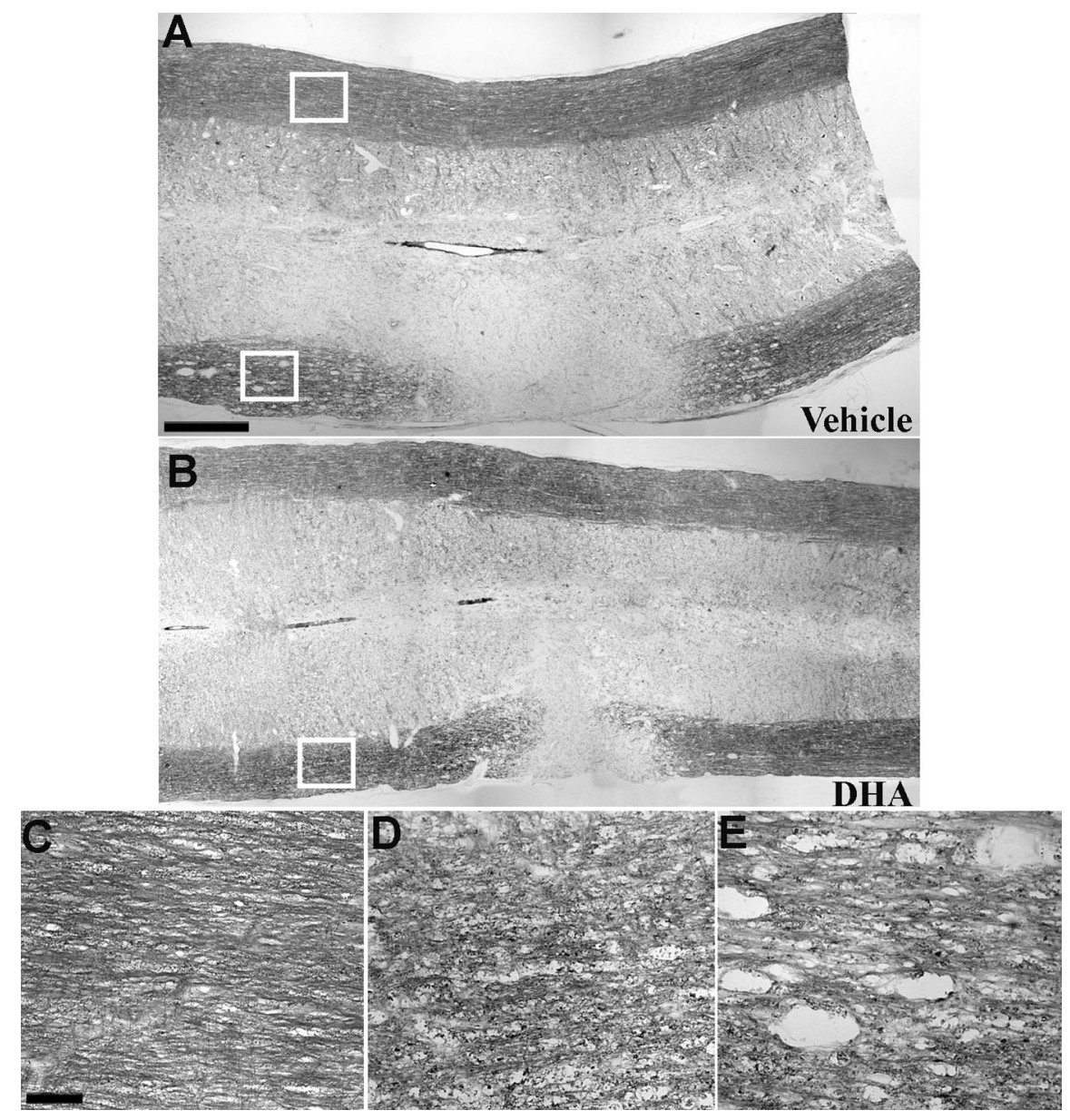

Figure 5. Luxol fast blue staining of the lateral whiter matter after spinal cord hemisection in animals that received a single intravenous injection of either saline or PUFAs after $7 \mathrm{~d}$ of survival. The appearance of myelin staining at the lesion site in vehicle-treated $(\boldsymbol{A})$, DHA-treated $(\boldsymbol{B})$, and ALA-, OA-, and AA-treated (not pictured) animals was similar, with all groups showing a lack of staining in the white matter ipsilateral to the lesion. Adjacent to the lesion site, the myelin in the white matter ipsilateral to the hemisection was disrupted relative to the uninjured side ( $\boldsymbol{C}$, enlargement of area within the top box in $\boldsymbol{A}$ ) in all groups. However, myelin in ALA-treated (not pictured) and DHA-treated ( $\boldsymbol{D}$, enlargement of area within the box in $\boldsymbol{B}$ ) animals was clearly less disrupted compared with $0 A-, A A-$, and vehicle-treated animals ( $\boldsymbol{E}$, enlargement of area within the bottom box in $\boldsymbol{A})$, which had large cavities in the white matter several millimeters from the lesion site. Scale bars: (in $\boldsymbol{A}) \boldsymbol{A}, \boldsymbol{B}, 250 \mu \mathrm{m}$; (in $\boldsymbol{C}$ ) $\boldsymbol{C}-\boldsymbol{E}, 25 \mu \mathrm{m}$.

models of neurotoxicity (Zararsiz et al., 2005) and forebrain ischemia (Blondeau et al., 2002; Cao et al., 2004) but also attenuate decreases in the CNS levels of antioxidant enzymes (e.g., catalase, superoxide dismutase, and glutathione peroxidase) in these models. To more clearly address the antioxidant role of DHA treatment, studies in our laboratory examining the effects of DHA treatment after spinal cord injury on both lipid peroxidation and protein oxidation are currently underway. It should also be noted that ALA and DHA may mediate their neuroprotective effects by reducing glutamate-induced excitotoxicity. In vitro and in vivo studies have shown that $\omega$-3 PUFAs reverse glutamateinduced excitotoxicity (Hogyes et al., 2003; Wang et al., 2003), which is known to be one of the triggers of apoptosis after SCI (Xu et al., 2004). Similar mechanisms may also underlie the protective effects of $\omega$-3 PUFAs on oligodendrocytes, because excitotoxicity also plays a role in their death (Park et al., 2004).

The striking difference in the neuroprotective efficacy of the $\omega-3$ and $\omega-6$ PUFAs seen in the current study also implicate effects on the inflammatory cascade that occurs after SCI. AA is metabolized by cyclooxygenase (COX) enzymes to two-series prostaglandins and thromboxanes, such as prostaglandin $\mathrm{E}_{2}$ and by 5 -lipoxygenase to produce leukotrienes such as leukotriene $\mathrm{B}_{4}$. These metabolites enhance vascular permeability, increase local blood flow, increase infiltration of leukocytes, and enhance production of proinflammatory cytokines such as tumor necrosis factor $\alpha$, interleukin 1 (IL-1) and IL-6. In contrast, $\omega$-3 PUFAs can decrease COX activity and the formation of proinflammatory eicosanoids and cytokines (Calder, 2003; Lonergan et al., 2004). SCI results in an increase in tissue AA concentration of up to 20 times (Murphy et al., 1994), and recruitment of neutrophils, macrophages, and microglial cells at the injury site (Dusart and Schwab, 1994). Antagonism of this deleterious cascade may therefore have contributed to the neuroprotective effects of DHA. In contrast, acute AA administration after injury could be expected to augment inflammation and increase cell death, as observed in our study.

It should be noted that the neuroprotective effects of the two tested $\omega$-3 PUFAs (ALA and DHA) may be mediated, at least in part, by different mechanisms. The finding that only $0.2 \%$ of ALA is converted to DHA in the rat suggests that the neuroprotective effect of ALA is not simply a result of its conversion into DHA (Demar et al., 2005). A direct neuroprotective mechanism of action could involve twopore potassium channels, such as TWIKrelated potassium channel (TREK) and TWIK-related arachidonic acidstimulated potassium channel (TRAAK), at which ALA and DHA have significant affinity (Lauritzen et al., 2000; Emsley et al., 2003). In addition, it has been shown that DHA can lead to the formation of mediators such as 10,17S-docosatriene (neuroprotectin D1), an endogenous compound with anti-oxidant (Bazan, 2005) as well as anti-inflammatory (Mukherjee et al., 2004) effects. ALA is converted in vivo to EPA, which is a precursor of the anti-inflammatory series-3 prostaglandins and also inhibits conversion of AA to proinflammatory prostaglandins and leukotrienes (Needleman et al., 1979). Recent pilot data in our laboratory show that EPA is neuroprotective in SCI and support the possibility that some of the effects of DHA are caused by conversion to EPA. Finally, the neuroprotective effect of DHA may also involve a complex pattern of activation of gene transcription, for example through retinoid receptor signaling (Jump, 2002). We have not yet performed detailed pharmacokinetic studies, and we have not analyzed the incorporation of the PUFA in the nervous tissue after the intravenous bolus injection in the rat, but by analogy with what has been reported in studies with radiolabeled AA (Jones et al., 1996), it is likely that a fraction of the injected DHA entered the CNS $(<1 \%$ reported for AA in animals with an intact blood-brain barrier) and was incorporated rapidly in phospholipids. We injected a bolus of DHA solution at $62.5 \mu \mathrm{M}$, and the free DHA in rat plasma is reported to be $10.6 \mu \mathrm{M}$, whereas the free DHA in brain is $\sim 1.3 \mu \mathrm{M}$ (Calderon 

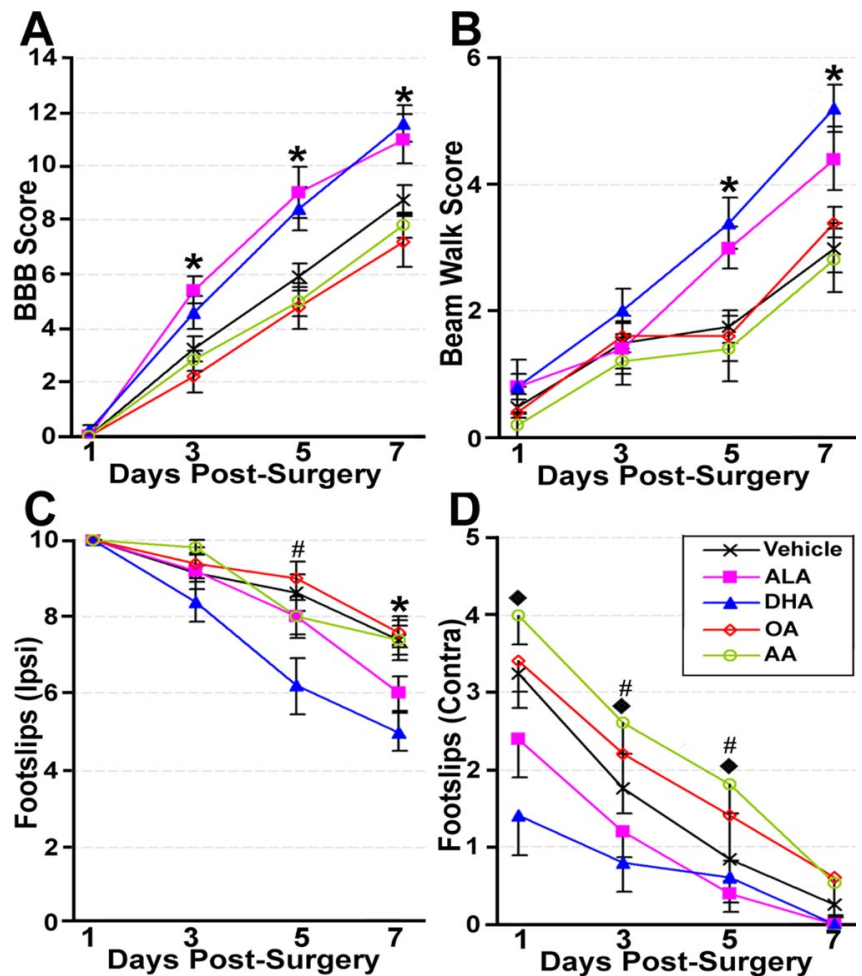

Figure 6. Locomotor performance after spinal cord hemisection in animals that received a single intravenous injection of either saline or PUFAs. Both ALA- and DHA-treated animals performed significantly better on the BBB open field $(\boldsymbol{A})$ and the beam walk $(\boldsymbol{B})$ tasks than the remaining groups, which did not differ. DHA- and ALA-treated animals also had significantly fewer foot slips with the hindlimb ipsilateral to the lesion on the horizontal ladder ( $\boldsymbol{C}$ compared with the other groups, although this difference was only seen for the ALA group at $7 \mathrm{~d}$ after surgery. In contrast, DHA- but not ALA-treated animals showed significantly fewer slips with the hindlimb contralateral to the lesion, compared with vehicle- and OA-treated animals, whereas AA-treated animals were worse on this parameter than all of the other groups $(\boldsymbol{D})$. *DHA- and ALA-treated animals significantly different from remaining groups ( $p<0.05$ ); \#DHA-treated animals significantly different from the remaining groups $(p<0.05)$; vehicle-, DHA-, and ALA-treated animals significantly different from AA-treated animals $(p<0.05)$.

and Kim, 2004). Therefore, the concentrations of DHA remained in a physiological range and were also compatible with the affinity of this fatty acid for targets such as TRAAK/TREK or retinoid receptors, which may be activated by the free DHA, thus being mechanistically involved in the neuroprotection seen.

The decreased lesion size and cell death obtained with ALA and DHA were mirrored by improved locomotor performance. This was seen in both a more rapid recovery and improved level of locomotor performance. This very close correspondence between the histological and behavioral parameters was seen as late as 6 weeks after spinal cord compression and means that it is highly likely that the functional improvements seen with $\omega$-3 PUFAs are a direct consequence of the decreased lesion size and decreased cell death. The large increase in the number of NeuNlabeled neurons seen after $\omega$-3 treatment in both injury paradigms suggests that this reduced secondary degeneration reflects increased survival of neurons. However, our data on oligodendrocyte survival and myelin integrity (Luxol fast blue) would indicate that it is also likely to reflect reduced damage to ascending and descending tracts. It is also important to note that $\omega$-3 fatty acids have been shown to have neurite growth-promoting effects in hippocampal neurons (Calderon and Kim, 2004). We recently confirmed such trophic effects in our laboratory, in stud-

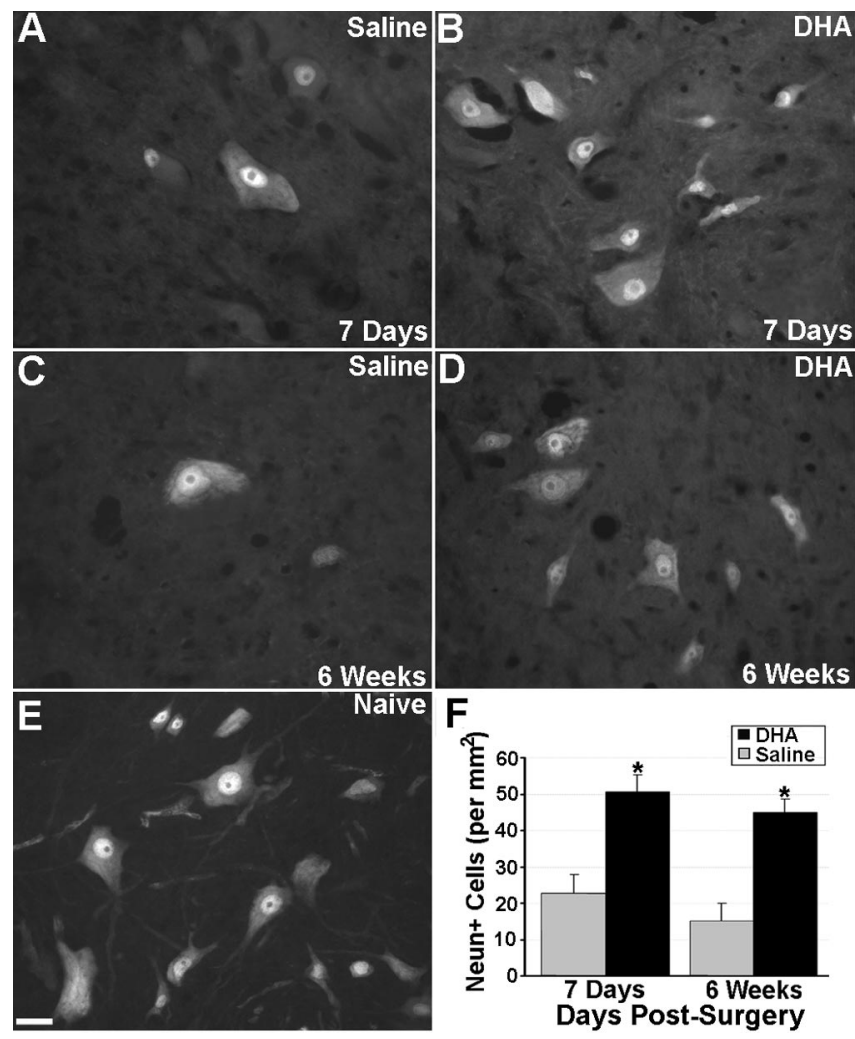

Figure 7. NeuN labeling of neurons after spinal cord compression and a single intravenous injection of either DHA or saline. At 1 week after surgery, the number of NeuN-labeled cells in saline-treated animals $(\boldsymbol{A})$ was substantially less than naive control animals $(\boldsymbol{E})$. DHA-treated animals $(\boldsymbol{B}, \boldsymbol{D})$ also had more NeuN-labeled neurons compared with saline-treated $(\boldsymbol{A}, \boldsymbol{C})$ animals at both 1 and 6 weeks after surgery. Quantitative analysis $(\boldsymbol{F})$ confirmed that there were significantly more NeuN-labeled neurons in DHA-treated animals at both 1 week and 6 weeks after surgery compared with saline-treated controls. Scale bar: (in $\boldsymbol{E}) \boldsymbol{A}-\boldsymbol{E}, 50 \mu \mathrm{m}$. *DHAtreated animals significantly different from saline-treated controls $(p<0.05)$. Error bars represent SEM.

ies on primary sensory neurons in culture (L. G. Robson, D. Sidloff, S. C. Dyall, and A. T. Michael-Titus, manuscript in preparation). Omega-3 compounds may therefore have not only neuroprotective but also neuroregenerative effects after SCI.

Together, our data suggest that $\omega$-3 PUFAs have significant therapeutic potential in SCI and are consistent with results with these PUFAs in other models of CNS injury (Blondeau et al., 2002; Cao et al., 2004; Zararsiz et al., 2005). However, some issues deserve additional investigation. For example, before recommendations for clinical use, the choice of compound and dose must be further explored and optimized. The dose of DHA we injected intravenous ( $250 \mathrm{nmol} / \mathrm{kg}$; i.e., $82 \mu \mathrm{g} / \mathrm{kg}$ ) is very small compared with the doses of EPA and DHA taken orally by patients in fish oil-containing preparations (e.g., 3-6 g/d) or compared with the intake of DHA as part of lipid preparations administered parenterally in the clinic (e.g., $2.15 \mathrm{~g}$ of DHA/100 $\mathrm{ml}$ in fish oilenriched suspensions) (Mayser et al., 2002). Therefore, this neuroprotective dose range could be easily translated to the clinic for use as an intravenous bolus after trauma. Heurteaux et al. (2005) recently showed that ALA treatment can be delayed up to $3 \mathrm{~h}$ and still induce significant neuroprotection after cerebral ischemia in the mouse. It is therefore important to determine whether similar (or longer) delays in $\omega-3$ PUFA treatment after SCI still confer neuroprotection. Similarly, the route of administration of these compounds after injury is of interest for their clinical application. 


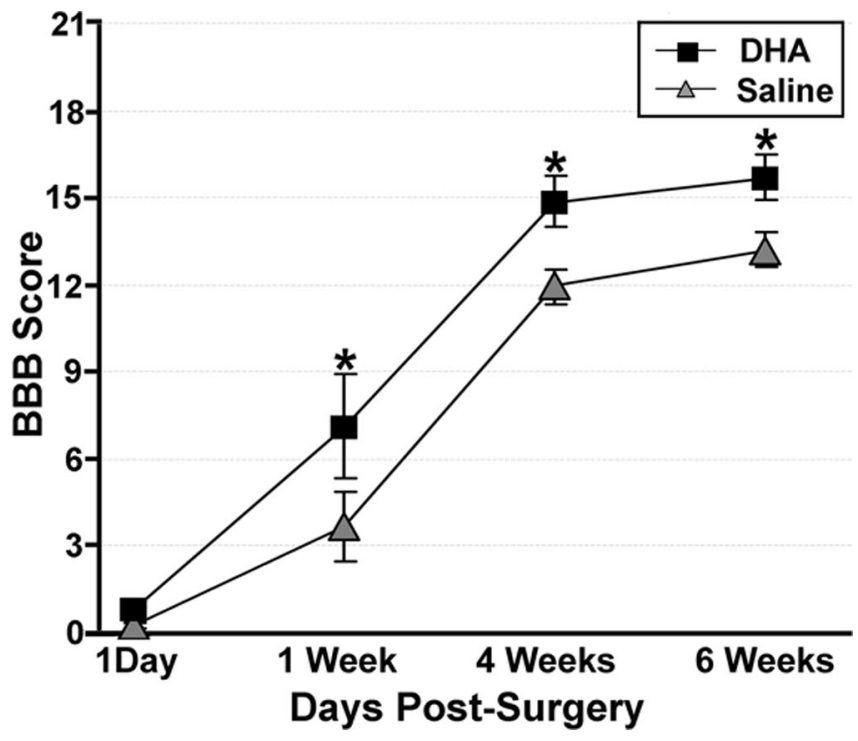

Figure 8. Locomotor performance of animals that received a spinal cord compression injury and a single intravenous injection of either DHA or saline. Both DHA- and saline-treated animals showed similar low levels of locomotor function $1 \mathrm{~d}$ after surgery as assessed on the $B B B$ open field task. However, by 1 week after surgery, DHA-treated animals show significantly better locomotor function (3 points on the BBB scale) compared with saline-treated animals, a difference that was maintained until 6 weeks after surgery (the latest point examined). ${ }^{*}$ DHA-treated animals significantly different from saline-treated controls $(p<0.05)$.

Initial evidence indicates that $\omega$-3 PUFAs are also neuroprotective after spinal cord injury when given via the diet (Huang et al., 2005). This is particularly relevant for the clinical situation, in light of the current use of lipid emulsions for nutritional support in a variety of patient populations, including surgical and critically ill patients (Calder, 2003).

Omega-3 PUFA preparations have been shown previously to be safe and well tolerated in patients in several diseases (Schlanger et al., 2002), which reinforces their potential for rapid translation into clinical use in SCI. Their significant potential in neurology is demonstrated by the remarkable efficacy of DHA in Zellwegger's syndrome (Martinez et al., 2000). In addition, as noted above, lipid emulsions are already given as nutritional support to a variety of vulnerable patient populations (Calder, 2003). However, most preparations used at present contain a significant amount of soybean oil and therefore have a considerable $\omega-6$ component that, according to our results with AA, could worsen outcome after SCI. In contrast, acute $\omega$-3 PUFA administration after trauma as a neuroprotective treatment would confer clear advantages and deserves consideration as a promising innovative approach in SCI management, with easy transfer to the clinic.

\section{References}

Basso DM, Beattie MS, Bresnahan JC (1996) Graded histological and locomotor outcomes after spinal cord contusion using the NYU weight-drop device versus transection. Exp Neurol 139:244-256.

Bazan NG (2005) Neuroprotectin D1 (NPD1): a DHA-derived mediator that protects brain and retina against cell injury-induced oxidative stress. Brain Pathol 15:159-166.

Beattie MS, Farooqui AA, Bresnahan JC (2000) Rev of current evidence for apoptosis after spinal cord injury. J Neurotrauma 17:915-925.

Blondeau N, Widmann C, Lazdunski M, Heurteaux C (2002) Polyunsaturated fatty acids induce ischemic and epileptic tolerance. Neuroscience 109:231-241.

Calder PC (2003) Long-chain n-3 fatty acids and inflammation: potential application in surgical and trauma patients. Braz J Med Biol Res 36:433-446.
Calderon F, Kim HY (2004) Docosahexaenoic acid promotes neurite growth in hippocampal neurons. J Neurochem 90:979-988.

Cao DH, Xu JF, Xue RH, Zheng WF, Liu ZL (2004) Protective effect of chronic ethyl docosahexaenoate administration on brain injury in ischemic gerbils. Pharmacol Biochem Behav 79:651-659.

Demar Jr JC, Ma K, Chang L, Bell JM, Rapoport SI (2005) Alpha-linolenic acid does not contribute appreciably to docosahexaenoic acid within brain phospholipids of adult rats fed a diet enriched in docosahexaenoic acid. J Neurochem 94:1063-1076.

Dusart I, Schwab ME (1994) Secondary cell-death and the inflammatory reaction after dorsal hemisection of the rat spinal-cord. Eur J Neurosci 6:712-724.

Emsley R, Oosthuizen P, van Rensburg SJ (2003) Clinical potential of omega-3 fatty acids in the treatment of schizophrenia. CNS Drugs 17:1081-1091.

Endres S, von Schacky C (1996) n-3 polyunsaturated fatty acids and human cytokine synthesis. Curr Opin Lipidol 7:48-52.

Facheris M, Beretta S, Ferrarese C (2004) Peripheral markers of oxidative stress and excitotoxicity in neurodegenerative disorders: tools for diagnosis and therapy? J Alzheimers Dis 6:177-184.

Hall ED, Braughler JM (1986) Role of lipid peroxidation in post-traumatic spinal cord degeneration: a review. Cent Nerv Syst Trauma 3:281-294.

Heurteaux C, Laigle C, Blondeau N, Jarretou G, Lazdunski M (2005) AlphaLinolenic acid and riluzole treatment confer cerebral protection and improve survival after focal brain ischemia. Neuroscience 137:241-251.

Hogyes E, Nyakas C, Kiliaan A, Farkas T, Penke B, Luiten PG (2003) Neuroprotective effect of developmental docosahexaenoic acid supplement against excitotoxic brain damage in infant rats. Neuroscience 119:999-1012.

Huang WL, Curran OE, Dyall S, George KJ, Priestley JV, Michael-Titus AT (2005) Docosahexaenoic acid exerts significant neuroprotection in a rat model of compression spinal cord injury. Soc Neurosci Abstr 31:672.9.

Huang WL, Robson D, Liu MC, King VR, Averill S, Shortland PJ, Priestley JV (2006) Spinal cord compression and dorsal root injury cause upregulation of activating transcription factor-3 in large-diameter dorsal root ganglion neurons. Eur J Neurosci 23:273-278.

Jones CR, Arai T, Bell JM, Rapoport SI (1996) Preferential in vivo incorporation of $[3 \mathrm{H}]$ arachidonic acid from blood in rat brain synaptosomal fractions before and after cholinergic stimulation. J Neurochem 67:822-829.

Jump DB (2002) Dietary polyunsaturated fatty acids and regulation of gene transcription. Curr Opin Lipidol 13:155-164.

Katsuki H, Okuda S (1995) Arachidonic acid as a neurotoxic and neurotrophic substance. Prog Neurobiol 46:607-636.

King VR, Phillips JB, Brown RA, Priestley JV (2004) The effects of treatment with antibodies to transforming growth factor betal and beta2 following spinal cord damage in the adult rat. Neuroscience 126:173-183.

Lang-Lazdunski L, Blondeau N, Jarretou G, Lazdunski M, Heurteaux C (2003) Linolenic acid prevents neuronal cell death and paraplegia after transient spinal cord ischemia in rats. J Vasc Surg 38:564-575.

Lauritzen I, Blondeau N, Heurteaux C, Widmann C, Romey G, Lazdunski M (2000) Polyunsaturated fatty acids are potent neuroprotectors. EMBO J 19:1784-1793.

Leker RR, Shohami E (2002) Cerebral ischemia and trauma-different etiologies yet similar mechanisms: neuroprotective opportunities. Brain Res Brain Res Rev 39:55-73.

Lonergan PE, Martin DS, Horrobin DF, Lynch MA (2004) Neuroprotective actions of eicosapentaenoic acid on lipopolysaccharide-induced dysfunction in rat hippocampus. J Neurochem 91:20-29.

Martin DS, Lonergan PE, Boland B, Fogarty MP, Brady M, Horrobin DF, Campbell VA, Lynch MA (2002) Apoptotic changes in the aged brain are triggered by interleukin-1beta-induced activation of $\mathrm{p} 38$ and reversed by treatment with eicosapentaenoic acid. J Biol Chem 277:34239-34246.

Martinez M, Vazquez E, Garcia-Silva MT, Manzanares J, Bertran JM, Castello F, Mougan I (2000) Therapeutic effects of docosahexaenoic acid ethyl ester in patients with generalized peroxisomal disorders. Am J Clin Nutr 71:376S-385S.

Mayser P, Mayer K, Mahloudjian M, Benzing S, Kramer HJ, Schill WB, Seeger W, Grimminger F (2002) A double-blind, randomized, placebocontrolled trial of $n-3$ versus n- 6 fatty acid-based lipid infusion in atopic dermatitis. J Parenter Enteral Nutr 26:151-158. 
Metz GA, Merkler D, Dietz V, Schwab ME, Fouad K (2000) Efficient testing of motor function in spinal cord injured rats. Brain Res 883:165-177.

Mukherjee PK, Marcheselli VL, Serhan CN, Bazan NG (2004) Neuroprotectin D1: a docosahexaenoic acid-derived docosatriene protects human retinal pigment epithelial cells from oxidative stress. Proc Natl Acad Sci USA 101:8491-8496

Murphy EJ, Behrmann D, Bates CM, Horrocks LA (1994) Lipid alterations following impact spinal cord injury in the rat. Mol Chem Neuropathol 23:13-26.

Needleman P, Raz A, Minkes MS, Ferrendelli JA, Sprecher H (1979) Triene prostaglandins: prostacyclin and thromboxane biosynthesis and unique biological properties. Proc Natl Acad Sci USA 76:944-948.

Nystrom B, Berglund JE, Bergquist E (1988) Methodological analysis of an experimental spinal cord compression model in the rat. Acta Neurol Scand 78:460-466.

Park E, Velumian AA, Fehlings MG (2004) The role of excitotoxicity in secondary mechanisms of spinal cord injury: a review with an emphasis on the implications for white matter degeneration. J Neurotrauma 21:754-774.

Profyris C, Cheema SS, Zang D, Azari MF, Boyle K, Petratos S (2004) Degenerative and regenerative mechanisms governing spinal cord injury. Neurobiol Dis 15:415-436.

Rodriguez-Rodriguez RA, Tabernero A, Velasco A, Lavado EM, Medina JM (2004) The neurotrophic effect of oleic acid includes dendritic differentiation and the expression of the neuronal basic helix-loop-helix transcription factor NeuroD2. J Neurochem 88:1041-1051.

Sarsilmaz M, Songur A, Kus I, Ozyurt B, Gulec M, Sogut S, Ilhan A, Akyol O (2003) The regulatory role of dietary omega-3 fatty acids on oxidant/ anti-oxidant balance in the rat hippocampus. Neurosci Res Commun 33:114-123.

Schlanger S, Shinitzky M, Yam D (2002) Diet enriched with omega-3 fatty acids alleviates convulsion symptoms in epilepsy patients. Epilepsia 43:103-104.

Songur A, Sarsilmaz M, Sogut S, Ozyurt B, Ozyurt H, Zararsiz I, Turkoglu AO (2004) Hypothalamic superoxide dismutase, xanthine oxidase, nitric oxide, and malondialdehyde in rats fed with fish omega- 3 fatty acids. Prog Neuropsychopharmacol Biol Psychiatry 28:693-698.

Sullivan PG, Rabchevsky AG, Waldmeier PC, Springer JE (2005) Mitochondrial permeability transition in CNS trauma: cause or effect of neuronal cell death? J Neurosci Res 79:231-239.

Toborek M, Malecki A, Garrido R, Mattson MP, Hennig B, Young B (1999) Arachidonic acid-induced oxidative injury to cultured spinal cord neurons. J Neurochem 73:684-692.

Unal-Cevik I, Kilinc M, Gursoy-Ozdemir Y, Gurer G, Dalkara T (2004) Loss of NeuN immunoreactivity after cerebral ischemia does not indicate neuronal cell loss: a cautionary note. Brain Res 1015:169-174.

Wang X, Zhao X, Mao ZY, Wang XM, Liu ZL (2003) Neuroprotective effect of docosahexaenoic acid on glutamate-induced cytotoxicity in rat hippocampal cultures. NeuroReport 14:2457-2461.

Xu GY, Hughes MG, Ye Z, Hulsebosch CE, McAdoo DJ (2004) Concentrations of glutamate released following spinal cord injury kill oligodendrocytes in the spinal cord. Exp Neurol 187:329-336.

Xu W, Chi L, Xu R, Ke Y, Luo C, Cai J, Qiu M, Gozal D, Liu R (2005) Increased production of reactive oxygen species contributes to motor neuron death in a compression mouse model of spinal cord injury. Spinal Cord 43:204-213.

Yong C, Arnold PM, Zoubine MN, Citron BA, Watanabe I, Berman NE, Festoff BW (1998) Apoptosis in cellular compartments of rat spinal cord after severe contusion injury. J Neurotrauma 15:459-472.

Zararsiz I, Kus I, Akpolat N, Songur A, Ogeturk M, Sarsilmaz M (2005) Protective effects of omega-3 essential fatty acids against formaldehydeinduced neuronal damage in prefrontal cortex of rats. Cell Biochem Funct, in press. 